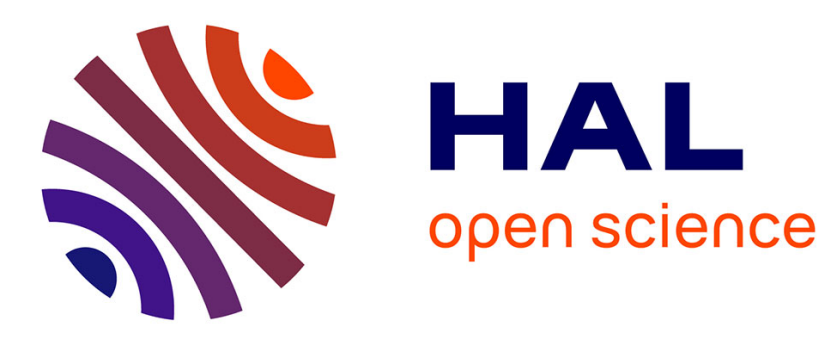

\title{
Aircraft deconfliction with speed regulation: new models from mixed-integer optimization
}

\author{
Sonia Cafieri, Nicolas Durand
}

\section{To cite this version:}

Sonia Cafieri, Nicolas Durand. Aircraft deconfliction with speed regulation: new models from mixedinteger optimization. Journal of Global Optimization, 2014, 58 (4), pp 613-629. 10.1007/s10898-0130070-1 . hal-00935215

\section{HAL Id: hal-00935215 \\ https://hal-enac.archives-ouvertes.fr/hal-00935215}

Submitted on 3 Apr 2014

HAL is a multi-disciplinary open access archive for the deposit and dissemination of scientific research documents, whether they are published or not. The documents may come from teaching and research institutions in France or abroad, or from public or private research centers.
L'archive ouverte pluridisciplinaire HAL, est destinée au dépôt et à la diffusion de documents scientifiques de niveau recherche, publiés ou non, émanant des établissements d'enseignement et de recherche français ou étrangers, des laboratoires publics ou privés. 


\title{
Aircraft deconfliction with speed regulation: new models from mixed-integer optimization
}

\author{
Sonia Cafieri ${ }^{1}$, Nicolas Durand ${ }^{1}$ \\ 1 École Nationale de l'Aviation Civile, 7 Ave. E. Belin F-31055 Toulouse France, \\ Email:sonia. cafieri@enac. fr, nicolas. durand@aviation-civile.gouv. fr
}

\begin{abstract}
Detecting and solving aircraft conflicts, which occur when aircraft sharing the same airspace are too close to each other according to their predicted trajectories, is a crucial problem in Air Traffic Management. We focus on mixed-integer optimization models based on speed regulation. We first solve the problem to global optimality by means of an exact solver. The problem being very difficult to solve, we also propose a heuristic procedure where the problem is decomposed and it is locally exactly solved. Computational results show that the proposed approach provides satisfactory results.
\end{abstract}

Keywords: air traffic management, conflict avoidance, MINLP, modeling, global exact solution, heuristic

\section{Introduction}

Aircraft sharing the same airspace are said to be potentially in conflict when they are too close to each other according to their predicted trajectories, that is, their relative horizontal and vertical distances do not both satisfy two given safety distances. Detection and resolution of aircraft conflicts, also referred to as aircraft deconfliction, is one of the most crucial issues in Air Traffic Management (ATM) to guarantee air traffic safety. This is even more evident observing that the air traffic level currently attained in Europe is around tens of thousands of flights per day and it is expected to be multiplied by a factor of two during the next 20 years [17]. The European project SESAR (Single European Sky ATM Research)[16] gives the guidelines to go towards an Air Traffic Management characterized by more efficiency and more safety, which should essentially result from a higher level of automation of ATM. The request for increasing automation of ATM comes from the observation that the high level of automation that has been introduced on aircraft in the last 50 years, for example with the Flight Management System, does not correspond to the level of automation on the ground. In the context of aircraft conflict detection and resolution, air traffic control is still widely performed manually on the ground by air traffic controllers watching the traffic movements on a radar screen and giving instructions to pilots. Therefore, the need for automatical tools to integrate human 
work, shifting responsibilities from the ground to the air, is evident. Increasing levels of traffic also raise the problem of managing traffic in such a way as to increase the capacity of control in the air sectors.

Aicraft potential conflicts can be solved in different ways, which lead to different mathematical modeling approaches. The most commonly exploited way is based on the idea of achieving separation changing the trajectory (heading) or the flight level of the aircraft involved in the conflict. This kind of separation maneuvers is the one usually exploited by air traffic controllers when they detect a potential conflict. Another way is based on the idea of separating aircraft by slightly changing their speeds but keeping the predicted trajectories. A speed regulation which occurs in a reasonable small range (namelly, from $-6 \%$ to $+3 \%$ of the original speed), allows a subliminal control as suggested by the European ERASMUS project [4]. This project showed the advantage of such a control, which is not perceived by air traffic controllers, and promoted it as an interesting alternative to the more traditional trajectory change.

Conflict avoidance is expected to be performed while deviating as little as possible from the original aircraft flight plan, that is, risks of collision should be avoided minimizing the impact of the separation maneuvers on the flight efficiency. To this aim, various solution strategies have been proposed for the corresponding optimization problem. A review is provided in [11]. Solution algorithms are mainly based on evolutionary computation $[8,5,7,6]$. Genetic algorithms tailored on the air traffic problem are widely used. These approaches consist in generating a population of aircraft trajectories from an initial population using the basic operators of selection, mutation and crossover, which are performed taking into account the features of the problem to gain efficiency. The solution space is a set of finite maneuvers, usually based on heading angle modifications. These methods are computationally efficient (few CPU time consuming), but the global optimal solution and even a feasible solution (with no conflicts) is not guaranteed to be achieved in a given time.

Recent advances in mixed-integer linear and nonlinear programming open new perspective for the deconfliction modeling and efficient solution. The first attempt to use mixed-integer optimization is by Pallottino et al. in [13], where, though under stringent hypothesis, an interesting modeling based on a geometrical construction leads to a mixed-integer linear programming problem that can be solved by CPLEX. More recently, mixed-integer programming has been proposed again for aircraft conflict resolution. In the very recent $\mathrm{PhD}$ thesis by Martin-Campo [12] (see also [1, 2]), new nonlinear models are proposed, starting from a modification of the one in [13]. In [18] a linear model is obtained for flight levels assignment and speed regulation for conflict avoidance. The speed regulation strategy is also modeled by mixed-integer programming in recent work $[14,15]$ for minimization of potential conflicts.

In this paper, we propose new modeling for aicraft deconfiction based on speed regulation, keeping trajectories unchanged. Mixed-Integer Nonlinear Programming (MINLP) formulations appear to be the natural candidates for the addressed ATM problems, where the need for modeling logical choices suggests the simultaneous presence of mixed 
(continuous-integer) variables, and nonlinear constraints arise from separation condition modeling. In this context, the main challenge is to propose mathematical formulations which are able to model the complex choices characterizing the target problems without imposing any unrealistic constraint.

We first compute deterministic global solutions, using a general-purpose solver for MINLP. To deal with the computational difficulty of the problem, we also propose another strategy, where the optimality guarantee is forsaken in exchange for the computational efficiency, but exact solutions are computed locally. This solution strategy is based on hybridizing mathematical programming and a heuristic tailored on the problem.

The paper is organized as follows. In Section 2 we propose two mixed-integer optimization models for aircraft deconfliction based on speed regulation. Section 3 deals with the computational solution of the considered problem. In subsection 3.2 we discuss the results of computational experiments carried out with a general purpose global optimization solver. The proposed heuristic based on local exact solutions is presented in subsection 3.3. Some concluding remarks are given in Section 4.

\section{Modeling aircraft deconfliction}

In a mathematical programming model for deconfliction, the main constraint is represented by the aircraft separation condition. We therefore first discuss about such condition. We assume, for the sake of simplicity, that aircraft fly at the same flight level. They are then identified by 2-dimensional points on a plane and the horizontal separation has to be respected. The aircraft separation between two aircraft $i$ and $j$ at the instant time $t$ is expressed by the following condition:

$$
\left\|\mathbf{x}_{i j}^{r}(t)\right\| \geq d
$$

where $d$ is the minimum required separation distance (usually, $5 \mathrm{NM}^{1}$ ) and $\mathbf{x}_{i j}^{r}(t)$ is the relative distance between aircraft $i$ and $j$.

Under the hypothesis that aircraft speed changes occur instantaneously at a given instant time $\bar{t}$, we can consider that uniform motion laws apply before and after $\bar{t}$. Hence, the relative position of aircraft $i$ and $j$ can be expressed as the sum of the relative initial position of aircraft $i$ and $j$ and the product of their relative speed $\mathbf{v}_{i j}^{r}$ by the time:

$$
\mathbf{x}_{i j}^{r}(t)=\mathbf{x}_{i j}^{r d}+\mathbf{v}_{i j}^{r} t \quad \forall t
$$

Squaring (1), with $\mathbf{x}_{i j}^{r}(t)$ expressed by (2), and deriving with respect to $t$, one can see that the minimum is attained for

$$
t_{m}=-\frac{\mathbf{v}_{i j}^{r} \mathbf{x}_{i j}^{r d}}{\left(v_{i j}^{r}\right)^{2}} .
$$

\footnotetext{
${ }^{1} 1 \mathrm{NM}($ Nautical Mile $)=1852 \mathrm{~m}$
} 
Substituting into (1), the following expression for the separation condition is obtained:

$$
\left(x_{i j}^{r d}\right)^{2}-\frac{\left(\mathbf{v}_{i j}^{r} \mathbf{x}_{i j}^{r d}\right)^{2}}{\left(v_{i j}^{r}\right)^{2}}-d^{2} \geq 0 .
$$

Note that condition (4) has to be checked only when the inner product $\mathbf{v}_{i j}^{r} \mathbf{x}_{i j}^{r d}$ is negative. In this case, indeed, aircraft are converging, generating a potential conflict.

A more classical approach consists in considering the separation constraint in the form

$$
\left\|\mathbf{x}_{i j}^{r}(t)\right\|=\left\|\mathbf{x}_{i}(t)-\mathbf{x}_{j}(t)\right\| \geq d \quad \forall t
$$

and discretizing with respect to the time variable $t$, obtaining a set of constraints each one corresponding to an instant time. Equation (4) does not depend on the time variable $t$ anymore, thus reducing the number of constraints. Nevertheless, in our modeling (4) is imposed in several time windows, as detailed in the following.

We now define mathematical programming models for the aircraft deconfliction problem such that aircraft separation is based only on speed regulation (other separation strategies, like heading angle or flight level modification, are not allowed). More precisely, starting from the known initial position and velocities of aircraft, reasonable small (in the sense that is precised below) changes in aircraft speeds are performed to have condition (4) satisfied along the predicted trajectories at each instant time $t$.

Various objective functions can be considered for the addressed problem. Conflict avoidance is expected to be performed while deviating as little as possible from the original aircraft flight plan, that is, risks of collision should be avoided minimizing the impact of the separation maneuvers on the flight efficiency. To stay as close as possible to the original flight plan, we minimize the sum of aircraft speed modifications:

$$
\min \sum_{k \in A} q_{k}^{2}
$$

where $\forall k$ in the set $A$ of aircraft, $q_{k}$ expresses the speed change of aircraft $k$. This function can be slightly modified to take into account the minimization of the time windows when the speed changes occur, as detailed below.

The decision variables are $q_{k}, k \in A$. These variables are bounded in order to ensure a "subliminal" speed control, as suggested by the ERASMUS project [4]. Specifically, we impose that the speed change for aircraft $k$ cannot be greater than $+3 \%$ and smaller than $-6 \%$ of its original speed. Other variables, both continuous and integer (in particular binary), are also employed in our problem formulation to express the afoarmentioned separation condition as follows.

In the first and simplest modeling we impose to aircraft an a priori speed change, at the instant time $t=0$, and let them keep the new speed (w.r.t. the original predicted one) during the whole trajectory. We introduce auxiliary continuous variables for the relative speed and the inner product $\mathbf{v}_{i j}^{r} \mathbf{x}_{i j}^{r d}$ in (4) and impose for each pair of aircraft $i$ and $j$ the separation condition (4). Equation (3) gives rise to a constraint, for each pair of aircraft, 
defining the minimum instant time. To check if $t_{m}$ is greater than 0 , a binary variable $y_{i j}$ is introduced for each pair $(i, j)$

$$
y_{i j}= \begin{cases}1 & \text { if } t_{m} \geq 0 \\ 0 & \text { otherwise }\end{cases}
$$

and constraints are added accordingly. The separation condition is then checked only when $t_{m} \geq 0$ :

$$
\forall i, j \in A \quad y_{i j}\left(\mathbf{x}_{i j}^{r d}\left(\mathbf{v}_{i j}^{r}\right)^{2}-\left(\mathbf{v}_{i j}^{r} \mathbf{x}_{i j}^{r d}\right)^{2}-\left(d^{2}\left(\mathbf{v}_{i j}^{r}\right)^{2}\right)\right) \geq 0
$$

We now propose a general model, where no conditions are imposed on the order and on the instant time of separation maneuvers execution. Each aircraft can modify its speed at any possible instant time $t_{s}$ during its trajectory and go back to its original speed at any possible instant time $t_{s}^{\prime}$. Instant times $t_{s}$ and $t_{s}^{\prime}$ are so unkown for each aircraft, and for each pair of conflicting aircraft it is not known the order of the respective instant times of speed change.

The main idea is then to deal with the different time windows where aircraft fly with their original (known) speed $v$ or with a changed speed $v+q, q$ representing a possible positive or negative speed change. Time windows are defined by instant times such that each aircraft changes its original velocity, i.e., it starts or ends flying with speed $v+q$. The speed is constant in each time window because of the assumption of instantaneous speed changes. In these time windows, aircraft $k$ flyes with speed $v_{k}$ or $v_{k}+q_{k}$. New decision variables are introduced, representing the instant times such that aircraft $k$ starts and respectively ends flying with changed speed:

$$
\forall k \in A \quad t_{1 k}, t_{2 k} .
$$

We have $t_{1 k} \leq t_{2 k} \forall k$. Furthermore, $t_{1 k}$ and $t_{2 k}$ are always $\geq 0$ and have an upper bound $T$, which represents the time horizon (usually around 20-30 minutes) during which the air traffic is observed and potential conflicts are solved. The optimization is performed repeatedly for successive time horizons with length $T$.

In order to be as close as possible to the original flight plans, the time intervals during which aircraft fly with a modified speed are also minimized. Thus, the objective function previously defined is modified in the following way:

$$
\min \sum_{k \in A} q_{k}^{2}\left(t_{2 k}-t_{1 k}\right)^{2}
$$

A number of auxiliary continuous and integer variables are also introduced. Suitable integer variables are in particular used to describe all possible time configurations.

Due to the fact that $t_{1 k}, t_{2 k} \forall k$ are unknowns of the problem and their sequential order is unknown as well, there are in fact 6 possible time configurations for each pair of aircraft. These are obtained considering the permutations of the 4 instant times characterizing the situation of a pair of aircraft changing their speed. Given a pair of aircraft $i$ and $j$, let 
$t_{1 i}, t_{1 j}$ and $t_{2 i}, t_{2 j}$ be the instant times when $i$ and $j$ start and respectively end flying with changed speed. An order for $t_{1 i}, t_{2 i}, t_{1 j}, t_{2 j}$ is not a priori known. By permutations of these instant times, excluding some cases giving rise to inconsistency (i.e., taking into account that $\forall k t_{1 k} \leq t_{2 k}$ and so a time sequence always starts with a $t_{1}$ instant and ends with a $t_{2}$ one), we obtain the following time configurations, where $T$ represents the upper bound on time instants:

$$
\begin{aligned}
& 0 \leq t_{1 i} \leq t_{1 j} \leq t_{2 i} \leq t_{2 j} \leq T \\
& 0 \leq t_{1 j} \leq t_{1 i} \leq t_{2 i} \leq t_{2 j} \leq T \\
& 0 \leq t_{1 i} \leq t_{2 i} \leq t_{1 j} \leq t_{2 j} \leq T \\
& 0 \leq t_{1 j} \leq t_{2 j} \leq t_{1 i} \leq t_{2 i} \leq T \\
& 0 \leq t_{1 i} \leq t_{1 j} \leq t_{2 j} \leq t_{2 i} \leq T \\
& 0 \leq t_{1 j} \leq t_{1 i} \leq t_{2 j} \leq t_{2 i} \leq T .
\end{aligned}
$$

It is easy to see, taking into account lower (0) and upper bound $(T)$ for instant times, that each of these configurations defines 5 time intervals.

Aircraft separation condition has to be imposed in each time interval, for each time configuration. A number of variables and constraints are so adjoined to the model to handle all possible configurations.

Firstly, the interval time for speed change must be at least equal to a certain amount $t_{\text {min }}$ :

$$
\forall k \in A \quad t_{2 k}-t_{1 k} \geq t_{\text {min }}
$$

To model all possible time configurations we introduce binary variables $z_{i j}^{\ell}, \ell \in\{1, \ldots, 6\}$, $i, j \in A$, stating, for each time configuration, what is the order of instant times for that configuration. For example, the binary variable $z_{i j}^{1}, i, j \in A$, associated to the first time configuration for the pair $(i, j)$, is such that:

$$
z_{i j}^{1}=\left\{\begin{array}{ll}
1 & \text { if } t_{1 i} \leq t_{1 j} \\
0 & \text { otherwise }
\end{array} \text { and } \quad t_{1 j} \leq t_{2 i} \quad \text { and } \quad t_{2 i} \leq t_{2 j}\right.
$$

The following constraint imposes that only one configuration must hold:

$$
\forall i, j \in A \sum_{\ell \in\{1, \ldots, 6\}} z_{i j}^{\ell}=1
$$

New variables are then introduced to define, for each pair of aircraft, their initial position in each time interval, their relative distance and their speeds. Distances covered by aircraft during each time interval are computed exploiting laws of uniform motion because of the aircraft's constant speed in each of such intervals. In the $h$-th time interval $\left[t_{s}, t_{s^{\prime}}\right], h \in\{1, \ldots, 5\}$, for all aircraft $k \in A$ the initial position $x_{k h}$ is given by

$$
x_{k h}=x_{k(h-1)}+\left(t_{s^{\prime}}-t_{s}\right) \bar{v}_{k},
$$


where $\bar{v}_{k}$ is the original speed $v_{k}$ or the changed speed $v_{k}+q_{k}$, depending on the time configuration holding. So, (continuous) variables $x_{k h} \forall k \in A \forall h \in\{1, \ldots, 5\}$, are introduced and corresponding constraints adjoined to the formulation, expressing for each aircraft the 5 initial positions in the 5 time intervals. Each aircraft $k$ has speed $\bar{v}_{k}$ equal to its original speed or to the changed speed depending on the time configuration, so that variables $z_{\ell}$ are used to identify the configuration holding. Relative distances $x_{i j}^{r d}$ and relative speeds $v_{i j}^{r}$ between aircraft are also defined, for each time configuration and each time interval, and constraints adjoined accordingly using variables $x_{k h}$ and again $z_{\ell}$.

Further constraints are then adjoined to the model to impose the condition (4) in each of the 5 time intervals, when $t_{m} \in\left[t_{s}, t_{s^{\prime}}\right]$, where $\left[t_{s}, t_{s^{\prime}}\right]$ is the $h$-th time interval. In order to check if $t_{m} \in\left[t_{s}, t_{s^{\prime}}\right]$, binary variables are used. For all $h \in\{1, \ldots, 5\}$ a binary variable $y_{l h}$ is introduced such that $y_{l h}=1$ if $t_{s h} \leq t_{m h}$ and 0 otherwise, $y_{r h}$ is such that $t_{m h} \leq t_{s^{\prime} h}$ and 0 otherwise. The following constraints are then imposed:

$$
\begin{aligned}
\forall h \in\{1, \ldots, 5\} & t_{s h} \leq t_{m h}+M\left(1-y_{l h}\right), & & t_{m h} \leq t_{s h}+M y_{l h} \\
& t_{m h} \leq t_{s^{\prime} h}+M\left(1-y_{r h}\right), & & t_{s^{\prime} h} \leq t_{m h}+M y_{r h}
\end{aligned}
$$

where $M$ is a sufficiently large constant. Condition (4) is then imposed for each time configuration $\ell \in\{1, \ldots, 6\}, \forall h \in\{1, \ldots, 5\}$ and $i, j \in A$, as follows:

$$
\left(y_{l h} y_{r h}\left(\left(x_{i j h}^{r d}\right)^{2}-\frac{\left(v_{i j h}^{r} x_{i j h}^{r d}\right)^{2}}{\left(v_{i j h}^{r}\right)^{2}}-d^{2}\right)\right) \geq 0 .
$$

Finally, for each time interval, the following separation condition is also imposed:

$$
\forall h \in\{1, \ldots, 5\}, \forall i, j \in A \quad\left(x_{i j h}^{r d}\right)^{2} \geq d^{2} .
$$

\section{Solving aircraft deconfliction}

\subsection{Test problems generation}

Let us consider as a testbed a set of $n$ aircraft in 2-dimensional space, placed on a circle of a given radius $r$, with initial speed $v$ and trajectory defined by a heading angle such that aircraft fly pointing toward the center of the circle (or slightly deviating with respect to such direction). The zone of conflict is around the center of the circle where aircraft are placed, and each aircraft may be in conflict with each other. In our computational experiments, we considered a time horizon $T=30$ minutes, the standard separation distance $d=5 \mathrm{NM}$, initial speed equal to $v=4 \times 10^{2} \mathrm{NM} / \mathrm{h}$ for all aircraft and heading angles randomly generated varying between \pm 5 degrees with respect to the trajectories pointing toward the center of the circle. All aircraft are in conflict with each other, so that a number of conflicts equal to $n(n-1) / 2$ is generated in the same conflict zone. See Figure 3.1. This kind of test problem, though does not correspond to a realistic situation, 
represents a good trade-off between simplicity of illustration and difficulty of resolution, to stress models and algorithms for deconfliction. It has been already used to this aim (see, e.g. [6]).

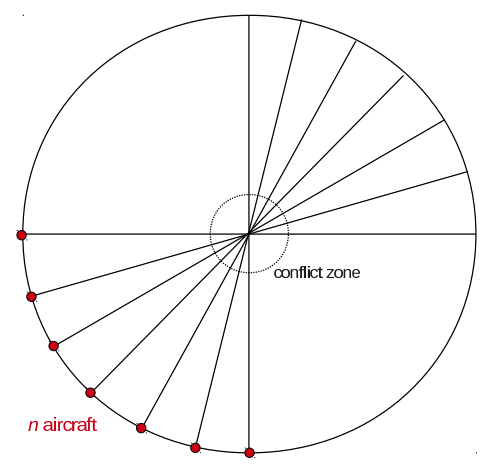

Figure 1: $n$ conflicting aircraft flying towards the center of a circle.

Results discussed in the following are obtained on a $2.4 \mathrm{GHz}$ Intel Xeon CPU of a computer with 8GB RAM shared by three other similar CPU running Linux.

\subsection{Global exact solution}

We implemented the proposed models using the AMPL modeling language [9].

We first employ a general purpose global optimization solver for MINLP to solve the addressed problem to global optimality. The solver of choice is COUENNE [3], which implements a spatial Branch-and-Bound based on convex relaxations. Results are reported in Table 1 for the first proposed model. For each problem instance (given by a number $n$ of aircraft and a radius $r$ of the circle representing the considered airspace) we report the objective function value and the CPU time to solve the problem. We are able to obtain global exact solutions up to $n=6$, i.e. 15 conflicts. Objective function values show that aircraft separation is always achieved with very slight speed changes.

\begin{tabular}{||c|c|c|c|c||}
\hline \hline$I D$ & $n$ & $r$ & obj & $\begin{array}{c}\text { CPU time } \\
\text { (sec.) }\end{array}$ \\
\hline pb_n2 & 2 & $1 \times 10^{2}$ & 0.002531 & 0.15 \\
pb_n3 & 3 & $2 \times 10^{2}$ & 0.001667 & 1.45 \\
pb_n4 & 4 & $2 \times 10^{2}$ & 0.004009 & 12.87 \\
pb_n5 & 5 & $3 \times 10^{2}$ & 0.003033 & 841.33 \\
pb_n6 & 6 & $3 \times 10^{2}$ & 0.006033 & 51863.37 \\
\hline \hline
\end{tabular}

Table 1: Conflict resolution for $n$ aircraft flying towards the centre of a circle of radius $r$ : objective function value and CPU time. Global optimal solutions are obtained with COUENNE.

Increasing the number of aircraft $n$, the number of variables, in particular of binary variables used to model logical choices, and the number of constraints largely increases, 
thus growing the size of the search tree to explore. Hence, the time to reach the leaves of the tree, which represent optimal solutions, may become very high. Hence, as expected for a Branch-and-Bound algorithm, high memory and time requirements do not allow us to obtain optimal solutions for larger dimensions of the problem.

Results in Table 1 refer to the relaxed modeling where we assume that aircraft change their speed at $t=0$ to achieve trajectory separation. The more general model that we propose in Sect.2 is, as expected, more computationally challenging, due specially to the larger number of binary variables and constraints used to model time configurations and time intervals. Therefore, we expect to solve to global optimality only small-size problems. For the sake of illustration, we describe computational results obtained with the most general model on problem instances involving a pair of aircraft. Results are given in Table 2. Aircraft are again supposed to move from an initial position given, in 2-dimensional space, on a circle $\left(r=1 \times 10^{2} \mathrm{NM}\right)$ with $v=4 \times 10^{2} \mathrm{NM} / \mathrm{h}$. Keeping the original speed, the aircraft would reach the center of the circle in 15 minutes. The problem is simplified in such a way that aircraft $k$ is assumed to change its speed on an instant $t_{1 k}$ and keep the new speed. For each instance we report data details, given by the heading angles of the two aircraft, and solution details, given by velocity change $\left(q_{k}\right)$ and instant time when the speed change occurs $\left(t_{1 k}\right)$ for aircraft $k$, objective function value and $\mathrm{CPU}$ time. Again, objective function values show that slight speed changes are sufficient to achieve separation. CPU times are less then 2 seconds, thus showing that even the general model is promising for optimal deconfliction in reasonable time for small-scale problems.

We note that symmetric equivalent solutions are possible for the considered test problem, where for pairs of aircraft we can swap their roles. Spatial Branch-and-Bound algorithms, like the one implemented in COUENNE, suffer from the presence of symmetries, which led to very large Branch-and-Bound trees. Simple symmetry breaking constraint like

$$
q_{i} \leq q_{j} \quad \forall i, j \in A, i<j,
$$

adjoined to the formulation, may help to speed up the resolution. However, they are not considered in our study, because they correspond to an unrealistic modeling of the addressed ATM problem.

\begin{tabular}{||c|c|c|c|c|c|c|c||}
\hline \hline \multicolumn{3}{|c|}{ aircraft 1 } & \multicolumn{3}{c||}{ aircraft 2} & obj & $\begin{array}{c}\text { CPU time } \\
\text { (sec.) }\end{array}$ \\
\hline heading & $q_{1}$ & $t_{11}$ & heading & $q_{2}$ & $t_{12}$ & & \\
\hline 0 & 0.943639 & 0.00611072 & $1 / 2 \pi$ & 1.0249 & 0.115235 & 0.000866779 & 1.99 \\
0 & -0.000001 & $3.84848 \mathrm{e}-7$ & $3 / 4 \pi$ & 0 & 0.373206 & $6.76119 \mathrm{e}-14$ & 0.08 \\
0 & 0 & 0.999991 & $5 / 4 \pi$ & -0.648046 & 0.0732228 & $1.59283 \mathrm{e}-11$ & 1.40 \\
\hline \hline
\end{tabular}

Table 2: Conflict resolution for 2 aircraft: optimal solutions and CPU time. The more general proposed model is used. 


\subsection{A heuristic based on local exact solutions}

From the numerical results discussed in the previous section, it appears that an exact solution algorithm for the addressed problem easily turns to be high memory and time demanding, due to the high number of conflicts and the number of variables and constraints largely increasing with $n$. However, we also note that on problem instances involving a small number of aircraft at a time, say 3-4 aircraft, a global solution can be efficiently computed. Based on this observation, we propose a solution procedure which is based on the idea of decomposing the overall problem into subproblems involving only a small number of aircraft and performing deconfliction on these subproblems, then combining all local solutions. The overall procedure is heuristic, based on Mathematical Programming and local exact solutions, in a matheuristic framework.

When dealing with aircraft conflict avoidance, one naturally looks at subproblems of the original one. In a realistic situation, the airspace section under consideration is a quite large portion of the airspace covering the trajectories of a large number of aircraft, among which generally only small groups of aircraft with close trajectories get potentially in conflict. This led some authors to introduce the concept of cluster.

Let a cluster be the transitive closing on conflicting pairs of aircraft (see [10]). The heuristic is based on the idea of solving the deconfliction problem on clusters involving up to 4 aircraft at a time. Let $n c l$ be the number of clusters. This number, and the aircraft therein, can be detected by a suitable pre-processing. The heuristic proceeds iteratively until deconfliction is successfully performed, or a given stopping criterion is satisfed. At each step, ncl deconfliction problems, one for each aircraft cluster, are sequentially solved by using an exact solver. Combining together all the results, one cannot expect in general to have all conflicts solved. This is because aircraft inside clusters may be in conflict also with aircraft inside other clusters. Should all conflicts be solved, the whole problem is solved and its solution is locally optimal. When this is not the case, that is, the number of remaining conflicts is greater than 0 , a new step is performed. The next iteration is made in such a way to preserve as much as possible the information outcoming from the previous solution. To do so, the initial speed (which together with the initial position represents the data of the problem) of aircraft that are still in conflict is re-initialized taking into account the solution obtained at the previous step. More precisely, if the (optimal) solution obtained for cluster $i$ is such that an aircraft in this cluster has been accelerated with respect to its original speed, then its speed is modified by a random slight further increase. If it has been decelerated, then its speed is modified by a random slight further decrease. In this way, the information obtained at the previous step is preserved and the chances to keep deconfliction inside clusters increase.

To update the aircraft speeds, a simple local search is performed. A number of candidates in the neighboroods of the initial speed values are tested, choosing the one that minimize a measure of the conflict severity. Specifically, we consider as such a measure the sum, over all conflicting aircraft, of the maximum violation of the separation constraints for each considered aircraft, relativized by dividing by the number of remaning conflicts. When one only conflict is to be solved, this search is intensified testing a larger number 
of candidates to increase the chances to solve the problem.

According to the ERASMUS directives, aircraft speed changes have to be bounded in the small range $[-6 \% v,+3 \% v], v$ being the original speed. To fulfill this requirement, when speeds are modified during the algorithm, these bounds are checked and speeds adjusted accordingly. This may eventually lead to change the speed scenario provided by local exact solutions.

A sketch of the proposed algorithm is given in Alg. 1. It may be intended as a general framework, where, starting from the same basic idea tailored on the problem, one can implement different local solution computations and local searches.

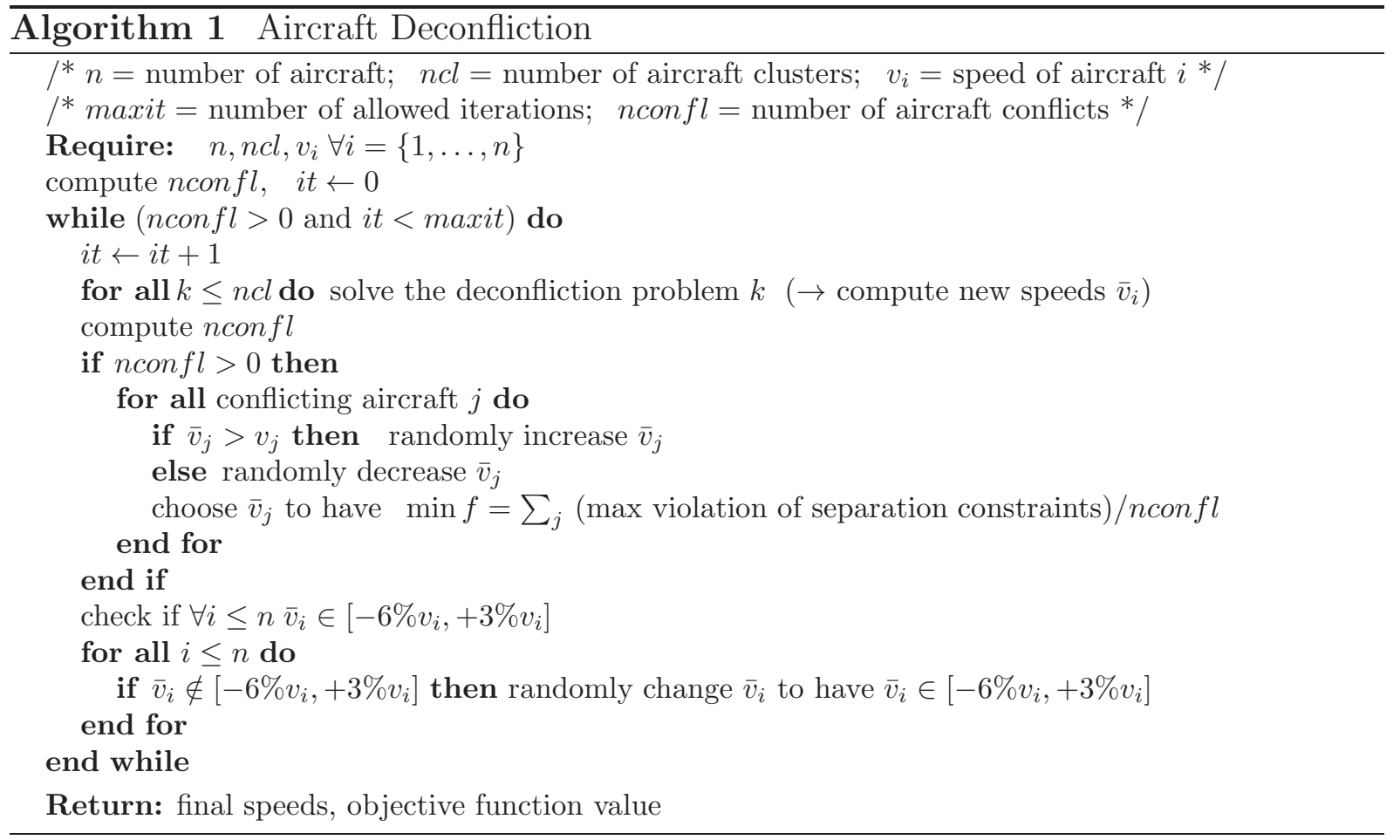

Results are reported in Table 3.3. Values are averaged over 10 runs. For all test problems, all conflicts have been solved $(n \operatorname{con} f l=0)$. Objective function values show that deconfliction is always achieved with very slight speed changes. Comparing the solutions obtained on pb1 using 1 cluster (direct solution on Table 1) or 2, it appears that decomposing the problem does not significantly affect the quality of the result. While for $n=4$ a direct solution is, as expected, more convenient, increasing $n$ faster solutions are obtained using a higher number of smaller subproblems. The sharp bound imposed on speeds changes to perform a subliminal control is responsible of the most of the execution time. This subliminal control is anyway performed in reasonable time even on problems involving many conflicts at a time. 


\begin{tabular}{||c|c|c|c|c|c||}
\hline \hline$I D$ & $n$ & $r$ & $n c l$ & $o b j$ & $\begin{array}{c}\text { CPU time } \\
\text { (sec.) }\end{array}$ \\
\hline pb_n4 & 4 & $2 \times 10^{2}$ & 2 & 0.005151 & 26.97 \\
pb_n5 & 5 & $3 \times 10^{2}$ & 2 & 0.004729 & 17.98 \\
pb_n6 & 6 & $3 \times 10^{2}$ & 2 & 0.006402 & 17.33 \\
pb_n6 & 6 & $3 \times 10^{2}$ & 3 & 0.007438 & 341.12 \\
pb_n7 & 7 & $3 \times 10^{2}$ & 2 & 0.009215 & 131.34 \\
pb_n7 & 7 & $3 \times 10^{2}$ & 3 & 0.008144 & 22.99 \\
pb_n8 & 8 & $4 \times 10^{2}$ & 2 & 0.008220 & 759.40 \\
pb_n8 & 8 & $4 \times 10^{2}$ & 3 & 0.007551 & 39.66 \\
pb_n8 & 8 & $4 \times 10^{2}$ & 4 & 0.012034 & 48.99 \\
pb_n9 & 9 & $4 \times 10^{2}$ & 3 & 0.009238 & 97.41 \\
pb_n10 & 10 & $4 \times 10^{2}$ & 3 & 0.014047 & 484.49 \\
\hline \hline
\end{tabular}

Table 3: Conflict resolution for $n$ aircraft flying towards the centre of a circle of radius $r$ : objective function value and CPU time. Solutions are obtained with the proposed heuristic beased on local exact solutions on ncl clusters.

\section{Conclusion}

We discussed about modeling and solving a challenging problem arising in Air Traffic Management, namely the problem of aircraft conflict resolution, via mixed-integer optimization. We proposed new MINLP formulations for deconfliction based on speed regulation, where conflicts are avoided allowing aircraft to only accelerate or decelerate, without any change in their trajectory. We first considered a speed regulation occurring at the instant time $t=0$, modifying the initial speed of aircraft with respect to the original predicted one. We then proposed a modeling where speed regulation occurs during time windows in such a way that aircraft fly with a modified speed in a time interval and then go back to their previous speeds. Speed changes and time intervals when they occur are minimized jointly.

We solved the problem using a general purpose deterministic global optimization solver, showing that mixed-integer optimization programs for aircraft deconfliction solved by deterministic global optimization provides promising results. We also proposed a heuristic tailored on the problem and based on locally solving the problem to global optimality, which provides good quality results for medium-scale problems.

Future work will address alternative mathematical programming formulations and possible reformulations of the proposed ones. From the point of view of solution approaches, a comparison with a Branch-and-Bound based on interval arithmetic and the further development of hybrid strategies, combining the above deterministic approaches with suitable heuristic ones, could provide interesting insights. 


\section{References}

[1] A. Alonso-Ayuso, L.F. Escudero, and F.J. Martn-Campo. Collision avoidance in air traffic management: a mixed-integer linear optimization approach. IEEE Transactions on Intelligent Transportation Systems, 12(1):47-57, 2011.

[2] A. Alonso-Ayuso, L.F. Escudero, and F.J. Martn-Campo. A mixed 01 nonlinear optimization model and algorithmic approach for the collision avoidance in ATM: Velocity changes through a time horizon. Computers and Operations Research, 2012. in press.

[3] P. Belotti, J. Lee, L. Liberti, F. Margot, and A. Wächter. Branching and bounds tightening techniques for non-convex MINLP. Optimization Methods and Software, 24(4):597-634, 2009.

[4] D. Bonini, C. Dupré, and G. Granger. How ERASMUS can support an increase in capacity in 2020. In Proceedings of the 7th International Conference on Computing, Communications and Control Technologies: CCCT 2009, Orlando, Florida, 2009.

[5] N. Durand and J.M. Alliot. Optimal resolution of en-route conflict. In Proceedings of the Eurocontrol/FAA ATM Seminar. Eurocontrol/FAA, 1997.

[6] N. Durand and J.M. Alliot. Ant-colony optimization for air traffic conflict resolution. In Proceedings of the Eighth USA/Europe Air Traffic Management Research and Development Seminar. Eurocontrol/FAA, 2009.

[7] N. Durand, J.M. Alliot, and F. Medioni. Neural nets trained by genetic algorithms for collision avoidance. Applied Artificial Intelligence, 2000.

[8] N. Durand, J.M. Alliot, and J. Noailles. Automatic aircraft conflict resolution using genetic algorithms. In Proceedings of the Symposium on Applied Computing, Philadelphia. ACM, 1996.

[9] R. Fourer and D. Gay. The AMPL Book. Duxbury Press, Pacific Grove, 2002.

[10] G. Granger and N. Durand. A traffic complexity approach through cluster analysis. In Proceedings of the 5th ATM REDD Seminar, Budapest, 2003.

[11] J. Kuchar and L. Yang. A review of conflict detection and resolution modeling methods. IEEE Trans. on Intelligent Transportation Systems, 1(4):179-189, 2000.

[12] F.J. Martin-Campo. The collision avoidance problem: methods and algorithms. $\mathrm{PhD}$ thesis,Rey Juan Carlos University, 2010.

[13] L. Pallottino, E. Feron, and A. Bicchi. Conflict resolution problems for air traffic management systems solved with mixed integer programming. IEEE Transactions on Intelligent Transportation Systems, 3(1):3-11, 2002. 
[14] D. Rey, S. Constans, R. Fondacci, and C. Rapine. A mixed integer linear model for potential conflict minimization by speed modulations. In Proceedings of the International Conference on Research in Air Transportation, Budapest, 2010.

[15] D. Rey, C. Rapine, R. Fondacci, and N-E. El Faouzi. Potential air conflicts minimization through speed regulation. Transportation Research Board, 2012.

[16] SESAR consortium: The european ATM master plan. Technical Report 1, European Commission and EUROCONTROL, 2009.

[17] Eurocontrol long-term forecast : IFR Flight Movements 2010-2030. Technical report, Eurocontrol - Air Traffic Statistics and Forecasts, 2010.

[18] A. Vela, S. Solak, W. Singhose, and J-P. Clarke. A mixed-integer program for flightlevel assignment and speed control for conflict resolution. In Proceedings of the joint 48th IEEE Conference on Decision and Control and 28th Chinese Control Conference, Shanghai. IEEE, 2009. 